\title{
I'DermoscopyApp: Hacking Melanoma thanks to IoT technologies
}

\author{
Giuseppe Di Leo, Consolatina Liguori, Antonio \\ Pietrosanto, Paolo Sommella \\ DIIn University of Salerno - Italy \\ tliguori@unisa.it
}

\author{
Vincenzo Paciello \\ DIEI, University of Cassino,- Italy \\ v.paciello@unicas.it
}

\begin{abstract}
The paper introduces I3DermoscopyApp, a new declination of the Internet of Things (IoT) paradigm, designed to allow the early detection of melanoma. Even though artificial intelligence programs cannot outperform the diagnostic accuracy of expert dermatologists yet, they reveal to be very useful in providing second opinions to physicians with short clinical experience, thus improving significantly their diagnostic performance. Following this trend, an original integration of mobile app technology and well-known image processing algorithms allows the automatic analysis of pigmented skin lesions to help physicians apply a diagnostic method (Seven Point Check List) based on dermoscopy. The web-based platform makes the physician able to: i) store digital images captured by smartphones featured with a dermatoscope; ii) measure morphological and chromatic parameters of the skin lesion; iii) make a diagnostic decision according to the Seven Point Checklist method. A detailed description of the adopted techniques, together with the first validation results are reported.
\end{abstract}

\section{Introduction}

Malignant melanoma is currently one of the leading cancers among many fair skinned populations around the world. In 2015 an estimated 73,870 new cases of invasive melanoma will be diagnosed in the US [1], where the overall 5-year survival rate for patients dramatically decreases (from $98 \%$ to $63 \%$ ) if melanoma is not detected before the tumor has spread to regional lymph nodes or other organs, also resulting in expansive (\$3.3 billion) annual cost for treating [2]. Consequently, in last two decades great attention was devoted to the early detection of melanoma through suitable information and screening campaigns as well as the technological developments [3] mainly addressed to the fields of imaging techniques [4] and diagnostic methods.
More in details, the Epiluminescence Microscopy (ELM, also known as dermoscopy) emerged as the preferred noninvasive tool for identifying subtle suspicious details and changes from the appearance of the skin lesion. It consist both of an optical system (as a magnification instrument) and an ad hoc light source designed to eliminate surface reflection and make the layers of the skin translucent, finally allowing a better visualization of several pigmented structures located within the epidermis, dermis and dermoepidermal junction. The addition of dermoscopy to routine melanoma screening typically reduces the proportion of cases submitted for excision biopsy (if compared with the naked-eye analysis by experienced examiners). Nevertheless, the diagnostic accuracy of dermoscopy depends on the amount of training of the dermatologist, the diagnostic difficulty of the lesions and the type of the algorithm/computerized tool used for assessment (the mainly adopted methods being based on Pattern Analysis, ABCDE rule, Seven and Three Point Checklist, Menzie scores). A very interesting summary about the dermoscopy and artificial intelligence for the diagnosis of melanoma is reported in [5]. As main findings of the systematic review (on more than 700 articles), there were no significant differences in diagnostic odds ratio among the dermoscopic algorithms, the pooled sensitivity for artificial intelligence was slightly higher than dermoscopy, whereas better results were achieved by dermoscopy in terms of pooled specificity. As a consequence, in the present form, the artificial dermoscopy [6] does not seem to increase the diagnostic accuracy of expert dermatologist, but it could be a diagnostic tool to be adopted by non-expert examiners, who typically exhibit poorer performance in terms of specificity [7]-[8].

In the authors' opinion, the artificial dermoscopy should provide useful information intended to $i$ ) suggest a second opinion to the dermatologist in the biopsy decision-making; and ii) train the inexperienced clinicians through identification and explanation (image highlighting) of the most complex and ambiguous morphological structures such as pigmented network, dots and globules within the lesion. In other 
words, to gain greater clinical acceptance, artificial intelligence (which is mainly focused on analysis and classification of low-levels feature and parameters, [9]) should be integrated/substituted by automatic detection systems providing higher level features (dermoscopic criteria) referred to a particular diagnostic scheme.

Following this approach, the problem of defining suitable image processing algorithm to implement the 7-Point Check List is pointed out. In [10] a complete tool for the automatic analysis of pigmented skin lesions is proposed whereas, in [11] the preliminary study on feasibility of a distributed Computer Aided System has been introduced.

In this paper, the authors are presenting I3DermoscopyApp, the web-based Application for melanoma automatic diagnosis. This App takes into account the work carried out to: i) analyze the patient perception of melanoma danger through interviews and social media; ii) involve the scientific dermatology community in a social-based approach to the diffusion of melanoma information; iii) introduce the uncertainty associated to the detection results as fundamental parameter in the decision making process.

The paper is organized as follows: in Section 2, a brief description of the outcomes of the HACKATHON about E-Health \& Innovation is reported; in Section 3, a system overview of the distributed application is described; in Section 4, some details about the image processing techniques and statistical approaches are presented; in Section 5, details about the automatic measurement and detection of dermoscopic structures are provided; in Section 6, experimental results about the validation of the measurement and e-learning application are reported; in Section 7, the conclusions are drawn.

\section{Hackathon on E-Health \& Innovation}

The research team was founded about 13 years ago and the experience and the research it has made in these years has taken the form of a software support for diagnosis (I3-DermoscopyApp). The last step of this long path was to share the project with those who can give a contribution, starting from the innovative point of view of different worlds that had rarely met before.

Hacking Melanoma (\#HackingMelanoma) has been an halfway between a traditional hackathon and a codesign event. Over 70 experts from computer hackers, engineers, top specialist dermatology and oncology, graduates, former patients and digital communication experts have been confronted for 36 hours non-stop analyzing eHealth scenarios and possible developments of the digital in research on melanoma [12].

Contamination among the different professionals involved has produced a high scientific value content, which in the following months have been organizing and disseminating, in order to develop a shared location on the topic with the rest of the scientific community, taking advantage of the potential that the Internet offers to competence networking. Among the outcomes of the Hackathon there is the analysis of the patient perception of the melanoma danger and a study about the sources which patients receive information from. Today HackingMelanoma is a scientific project that aims to rewrite the rules of scientific research on melanoma, breaking the traditional schemes.

\section{The Proposed Architecture}

An overview of the authors' proposal is depicted in Figure 1, where are highlighted the mobile app and the distributed platform which provides the remote service of automatic melanoma diagnosis.

The $I^{3}$ DermoscopyApp is the user front end developed for three different profiles and corresponding cases: 1) PATIENT, interested in achieving information about melanoma and specialized physicians; 2) PHYSICIAN, mainly interested in receiving the automatic (second) dermoscopic opinion about ambiguous skin lesions, and 3) top EXPERT dermatologist of the network, interested in maintaining the role of educational guidance and supervise the diagnostic process, by providing his evaluation of the skin lesion including the 7 Point Check List score (third opinion)

The App is designed to help professionals users acquire and store ELM images from smartphones and/or tablets featured with a dermatoscope, together with the patient personal information. To this aim, the sensitive records may be shared, stored in the cloud and managed by the company owner of the platform for 5 years, only with the aware consent by the patient about the adoption of personal information for research and educational purposes, according to Italian Privacy Act. About the security features, as a result of the hackathon, the encryption mechanism provided by the Https transmission has been considered as the minimum level to be assured in data communication.

The distributed platform includes two main components: the Web Server and the Application Engine. The former component, implemented as Apache Https Server, is designed to:

i) accept requests for lesion analysis from multiple users (step 1), by managing different queues corresponding to 3 main tasks: automatic image segmentation (step 2), store of experts' counseling (steps 6-7) and automatic lesion diagnosis (step 9);

ii) respond through dynamic web pages, by running suitable PHP scripts (steps 5, 13); and 
iii) manage a suitable database (as MySQL Manager) including lesion images and corresponding dermoscopic and clinical information in order to share the recorded data with the professional community (steps 4, 8, 11).

The Application Engine, which represents the automatic measurement and diagnosis System, performs the following operations:

i) Image Segmentation, allowing the pigmented lesion to be extracted from the surrounding healthy skin (step 3);

ii) Dermoscopic Structure Extraction and Classification, designed to identify and measure the main morphological and chromatic features of the lesion, necessary to their classification as ELM criteria (high-level structures) included in the 7-Point Checklist (step 10);

iii) Lesion Diagnosis and learning, providing the dermatologist with the automatic diagnosis (the lesion is classified as malignant melanoma, atypical nevus to

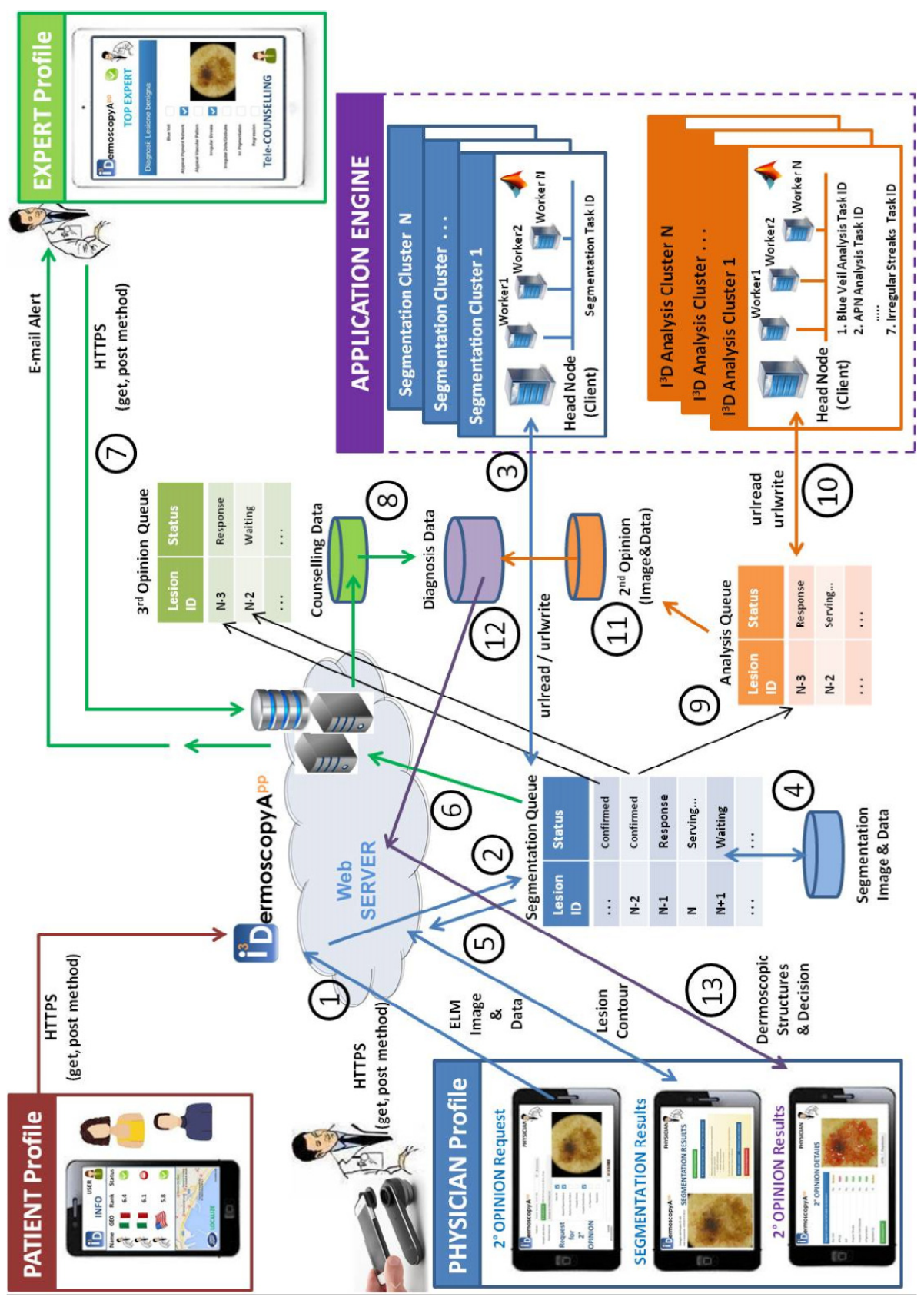

Figure 1. The proposed distributed framework for the Automatic Diagnosis of Melanoma. 
be excited, suspicious nevus to be followed-up or benign nevus) on the basis of a reliability based scoring (step 12). All the algorithms about the image preprocessing, the boundary extraction, the detection of low-level features and dermoscopic structures as well as the lesion classification are implemented as Matlab routines and executed as distributed code (jobs with multiple parallel tasks) by adopting the Parallel Toolbox and the built-in functions for the web communication with the Apache server. The third opinion, if available, is also included for learning purposes by top expert dermatologist.

A modular architecture is proposed that may encompass different multiple processing clusters both for redundancy (necessary to assure a continuous operation of the service) and scalability (in order to effectively match the expected growing of the market). Moreover, the modular architecture allows to enrich the measurement system with further software functionalities (as for example, clusters performing Pattern Matching with information already included in the Database, useful to improve the classification of new lesion and/or the presentation of the measurement results).

\section{Methods and Materials}

The Application Engine depicted in Figure 1 represents the integration of the most remarkable literature findings with the authors' proposal. The system has been applied (for training and testing) to a digital ELM image collection of over 600 skin lesions (partly extracted by the reference atlas [13] and partly captured during the clinical activity at the Dept. of Dermatology of the University Federico II, Naples, between 2008 and 2013). The system adopts different approaches and techniques [14] in terms of colour planes, mathematical operators, feature extraction methods, classification algorithms and statistical analysis.

\subsection{Image Segmentation}

At the Pre-processing stage, the hair removal algorithm (necessary to prevent the failure/degradation of the next lesion segmentation) has been implemented, following the approach suggested by Schmid in [15]. Then, an extensive statistical analysis is integrated into the automatic system, which includes the Adaptive Thresholding and the Statistical Region Merging (SRM), the most remarkable strategies for the boundary detection as reported in the comparisons [16],[10]. More in details, the former approach is suggested for negligible artifacts (see examples in Figure 2.a-b), whereas better results (closer to the expert dermatologists' indication) may be achieved by adopting SRM when extended black corners and oil bubbles are introduced during the image acquisition (see details reported in Figure 2.c-d).

\subsection{Feature Extraction and Classification}

Each (high-level) dermoscopic structure included in the 7-Point Checklist is automatically disclosed within the lesion through ad-hoc algorithms and/or statistical analysis, which take into account the image processing methods and techniques belonging to two macrocategories: color segmentation and structural analysis. The former group includes the adoption of both the $S R M$ and an alternative approach, proposed in [17] and investigated for dermoscopic images in [18], named Multi-Thresholding and based on the Principal Component Analysis (PCA, also known as the discrete Karhunen-Loeve Transform or Hotelling Transform [19]). Structural techniques designed to search for primitive structures such as points, lines and circles, have been extensively adopted for automatically detecting texture and/or local networks in dermoscopic images. Among these: a feature extraction based on the Laplacian of Gaussian (LOG) filtering [20], and the vessel enhancement filter, also known as Frangi's Filter [21].

As for classification methods, although a general model using supervised learning and Maximum A Posteriori Probability (MAP) estimation has been proposed [22] to perform common tasks in automated skin lesion diagnosis (also including border detection, artifact detection) with interesting and promising results, authors think that supervised learning could give better results in the final stage of feature classification. To this aim, the proposed automatic system encompasses the adoption of Machine Learning
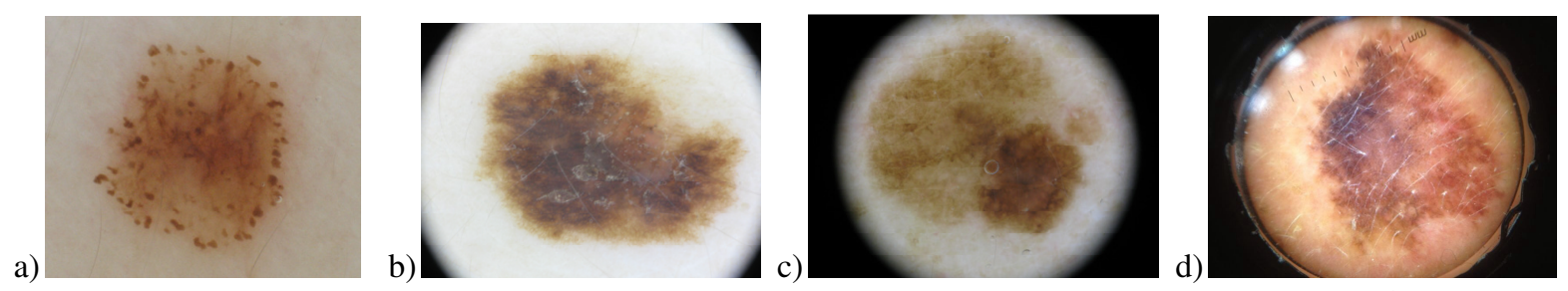

Figure 2. Boundary detection and Acquisition Artifacts: Adaptive Thresholding (a-b), SRM (c-d). 
a)

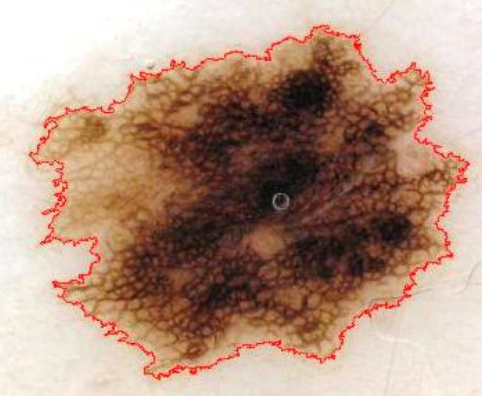

b)

c)
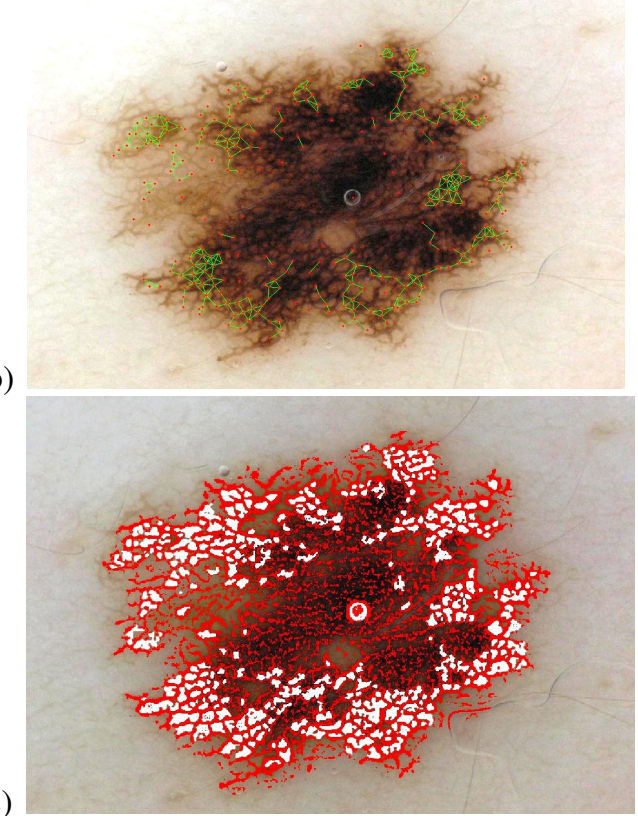

Figure 3. Detection Atypical Pigmented Network: a) lesion and superimposed contour;

b) result of the the Graph-based approach; c) highlight of the Atypical Pigmented Network (with High probability).

techniques [23]-[24] to solve the problem of data mining from the feature descriptors.

A well-known class is represented by the Decision Tree (DT) classifiers, firstly introduced in the computer-assisted analysis of ELM images in [25]. A DT classifier is a predictive model, trained (or induced) by adopting a suitable dataset with respect to which classification results are already available. The DT classifiers are generally preferred to other solutions (also including Artificial Neural Networks and Support Vector Machines, [9]) even if they usually exhibit high variance, because they are fast to train and apply and give low bias. Another popular method for classification is instead linear logistic regression.

In [18],[26], the SimpleLogistic classifier was proposed to perform the automatic detection of a)

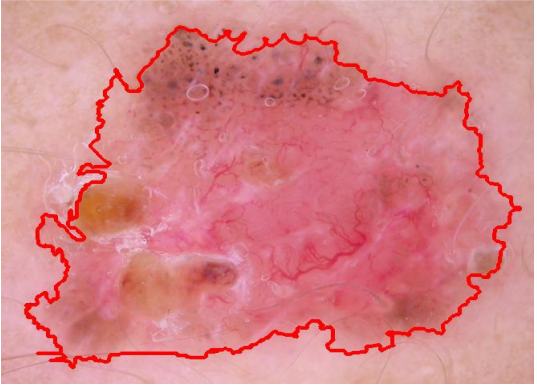

b)

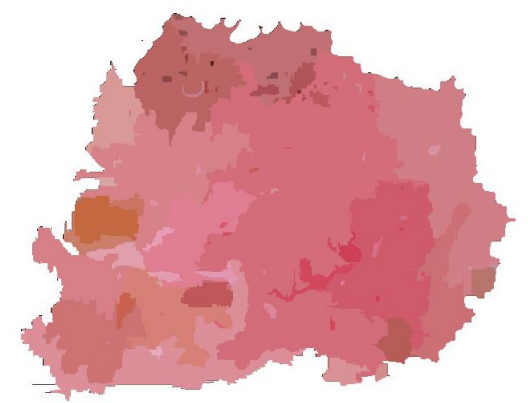

c)

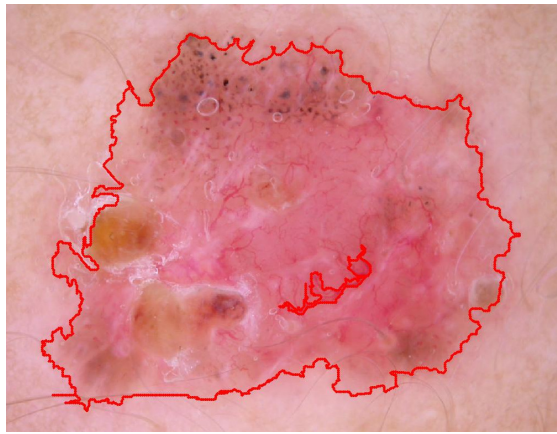

pigment network and irregular streaks respectively, through a process which was found to be quite stable, resulting in low variance but potentially high bias. Consequently, the way explored by the authors for performing the classification tasks has been a combination of a tree structure and logistic regression models resulting in a single tree according to the model proposed in [27]. Thus, the Logistic Model Tree (LMT) is often preferred for classifying the low-level features.

A complete summary of the image processing techniques and classification approach adopted for detecting the presence of the Dermoscopic criteria is reported in [11].

In addition, for each criterion detection, a statistical analysis has been performed (taking into account the results from the corresponding Training Set) in order to 
achieve a measurement information about the reliability of the adopted procedure. More precisely, to the detection of each dermoscopic structure a label (Low, Medium or High) is assigned according to different ranges for the corresponding metric value (used for qualifying the processing algorithm).

As an example, the LMT has been also trained to solve the three class problem (Absent /Typical/Atypical Classification) for the pigmented network within the lesion, by referring to the set of 69 structural, geometric and chromatic features suggested in [20] and extracted according to the Graph-based approach and the Iterative Loop Counting Algorithm (ILCA, [28]).

An example of the darker mesh of the pigmented network (referred to as the net) and the lighter colored areas the net surrounds (referred to as the holes) is depicted in Figure 3: the $P_{i}$ probabilities for the pigmented network is given by

$$
P_{i}=\frac{e^{F_{i}(x)}}{\sum_{j=1}^{2} e^{F j(x)}}
$$

with $i=1,2$ and 3 correspondingly respectively to the Atypical, Typical and Absent classes. The Atypical Pigmented Network is detected if:

$$
\arg \max \left(P_{i}\right)=1
$$

with a detection reliability labeled according to the following scheme:

$$
\begin{array}{lll}
\text { Low } & \text { if } & (0.34<) P_{1}<0.65 \\
\text { Medium } & \text { if } & 0.65 \leq P_{1}<0.85 \\
\text { High } & \text { if } & 0.85 \leq P_{1} \leq 1
\end{array}
$$

Another approach combining color segmentation and structural analysis is proposed for automatic detection of Atypical Vascular Pattern: the inner area of the lesion (see Figure 4.a-b) is considered and segmented through the Statistical Region Merging at fine level $(Q=256)$; then, the red segments are matched with the linear/globular structures selected from the enhanced image achieved by the tubularness filter response [21]. Finally, the candidate low-level features are associated to Atypical Vascular Pattern if their spatial distribution is not uniform with respect to the symmetry axes of the lesion (computed as the major and minor axis of the ellipse characterized by the same normalized second central moments as the region of interest). In the opposite case, i.e if the observed red linear/globular structures were randomly scattered within the lesion, the number of elements in each of 4 quadrants (as resulted from drawing the main lesion axes) could be modeled according to the Binomial Distribution [29].

Therefore, a Binomial Test can be performed to estimate the casual distribution of $N$ linear/globular objects, once the accepted risk $\alpha$ of Type I Error is fixed. According to the proposed approach, if the paucity or plenty of objects is observed in any quadrant and/or couple of quadrants, the Null Hypothesis is refused and the morphological structures are classified as Atypical Vascular Pattern with a detection reliability denoted as follows:

$\begin{array}{ll}\text { Low } & \text { if } N<15 \\ \text { Medium } & \text { if } 15 \leq N<30 \\ \text { High } & \text { if } \mathrm{N} \geq 30 .\end{array}$

Eq. (6)

Once the detection algorithms are performed and the corresponding reliability are computed, the lesion classification is carried out by considering a weighted version of the scoring system provided by the 7-Point Check List.
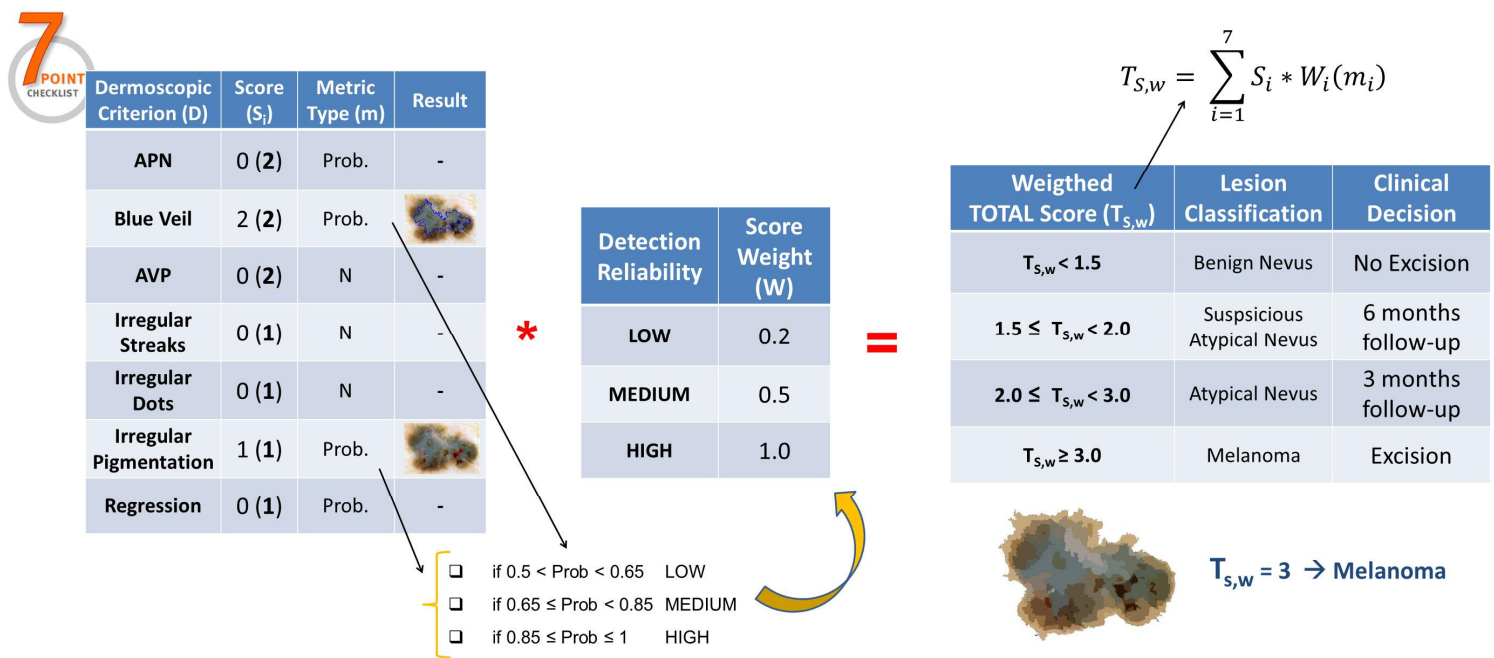

Figure 5. Application of the classification scheme for the clinical decision. 
Table 1. Automatic Classification according to the Weighted Scoring System.

\begin{tabular}{|c|c|c|}
\hline $\begin{array}{c}\text { Weighted } \\
\text { TOTAL Score }\end{array}$ & $\begin{array}{c}\text { Lesion } \\
\text { Classification }\end{array}$ & $\begin{array}{c}\text { Clinical } \\
\text { Decision }\end{array}$ \\
\hline$T_{S, w}<1.5$ & Benign nevus & No Excision \\
\hline $1.5 \leq T_{S, w}<2.0$ & $\begin{array}{c}\text { Suspicious } \\
\text { Atypical nevus }\end{array}$ & $\begin{array}{c}6 \text { months } \\
\text { follow-up }\end{array}$ \\
\hline $2.0 \leq T_{S, w}<3.0$ & Atypical nevus & Excision \\
\hline$T_{S, w}>3.0$ & Melanoma & Excision \\
\hline
\end{tabular}

Indeed, the partial scores are firstly weighted according to the detection uncertainty: three constant $(0.2,0.5$ and 1.0$)$ have been experimentally associated to the reliability ranges of the software routines (corresponding respectively to the LOW, MEDIUM and HIGH classes). Then, the seven weighted scores are summed-up to achieve a total score for the skin lesion, which is adopted to suggest the clinical decision (following-up or excision) according to the new scheme reported in Table 1. An example of the scheme application to the lesion image is depicted in Figure 5.

\section{Experimental Results}

The Application Engine has been run on a 64-bit Microsoft Windows system, featured with Intel Core i7 CPU at $2.67 \mathrm{GHz}$ and $6 \mathrm{~GB}$ RAM. The web platform has been adopted for collecting 270 ELM images as well as the corresponding dermoscopic information (patient age and sex, lesion position, first opinion from the physician and histological results after excision) during the screening campaign carried out from the Dept. of Systematic Pathology - Section of Dermatology at University "Federico II" of Naples, Italy.

The acquisition and transmission of the digital images have been performed through iPhone 4/5 smartphones equipped with two different types of dermoscope, namely the DermLite DL1 and Handyscope from FotoFinder GmbH. About the image quality, all the pictures were color images in JPEG format with dimensions ranging from $700 \times 447$ to $2272 \times 1520$ pixels. As a result of the a-posteriori analysis based on biopsy, the dermoscopic database included 64 melanoma, 99 atypical nevi (22 suspicious nevi) and 107 benign nevi.

With the aim to evaluate the measurement and diagnostic performance of the proposed platform, two different groups of dermatologists were asked to examine the dermoscopic database according to the Seven Point Checklist and provide the diagnosis for each lesion image: the former group (ED) included three physicians (B.B., S.C., M.A.) with more than 10 years' experience in dermoscopy, whereas the latter group (NED) included three young professionals (C.C., A.M., F.C.) with clinical activity $<3$ years.

The performance has been evaluated by distinguishing the diagnostic and clinical accuracy: the former value is computed as a weighted mean (according to the numerosity of the different classes) of sensitivity (ratio of the correct detection and the total number of melanoma) and specificity (ratio of the correct detection of benign/atypical nevi and the total number of lesions not classified as melanoma). The clinical accuracy instead takes into account the final decision of excision (both for melanoma and strongly atypical nevi) and following-up/ignoring (for benign nevi and lesions with few atypical structures).

In order to characterize the proposed routines for the automatic measurement of dermoscopic structures, the Seven Point Checklist scores by the expert dermatologist (S.C.) with the best exhibited diagnostic accuracy have been matched with the biopsy results, so that the detection reliability of the seven criteria have been achieved for each image included in the database. In details, every dermoscopic structure should be measured with high, medium or low probability if the lesion of interest is respectively classified as melanoma, atypical or suspicious nevus.

The Seven Point Checklist scores, diagnostic and clinical accuracy exhibited by the six dermatologists and the automatic measurement system (MS) are reported in Table 2.

As expected, the diagnostic and clinical accuracy of ED Group have been revealed as the best performance, with very high sensitivity and specificity (respectively around $80 \%$ and $90 \%$, typical values in other blind studies reported in literature). Also the clinical accuracy from the NED Group may be considered satisfying, thanks to the highest value for the exhibited specificity. Nevertheless, a different behavior is highlighted by the low sensitivity, which adversely affects the overall diagnostic accuracy. The lack of self-confidence in differentiating melanomas from strongly atypical lesions with respect to the expert dermatologists is also evident in the Seven Point Checklist scores. Whereas the major and minor criteria have been detected by the ED Group with good precision, showing a proper application of the dermoscopic algorithm (amount and distribution of the high level structures among the lesion classes reflect the score weight and diagnostic classification), the examiners included in the NED Group have been selected a greater number of both major and minor dermoscopic criteria without a clear association to the melanomas correctly detected. 
Table 2. Automatic Classification according to the Weighted Scoring System.

\begin{tabular}{|c|c|c|c|c|c|c|c|c|}
\hline & \multicolumn{8}{|c|}{ Examiner } \\
\hline & \multicolumn{3}{|c|}{ ED } & \multicolumn{3}{|c|}{ NED } & \multirow{2}{*}{ MS } & \multirow{2}{*}{$\begin{array}{l}\text { Biopsy/ } \\
\text { Reference }\end{array}$} \\
\hline & S.C. & B.B. & M.A. & C.C. & A.M. & F.C. & & \\
\hline Atypical Pigment Network & 86 & 71 & 85 & 93 & 89 & 106 & 92 & 72 \\
\hline Blue-whitish Veil & 54 & 32 & 47 & 105 & 74 & 35 & 58 & 53 \\
\hline Atypical vascular pattern & 15 & 28 & 12 & 19 & 37 & 18 & 28 & 18 \\
\hline Irregular streaks & 24 & 11 & 16 & 18 & 19 & 23 & 32 & 23 \\
\hline Regression structures & 74 & 15 & 76 & 66 & 35 & 91 & 87 & 68 \\
\hline Irregular Pigmentation & 74 & 53 & 56 & 122 & 114 & 103 & 46 & 65 \\
\hline Irregular Dots/Globules & 60 & 51 & 55 & 70 & 71 & 63 & 80 & 53 \\
\hline $\begin{array}{r}\text { Correct (Classified) } \\
\text { Melanoma }(M N)\end{array}$ & $59 / 69$ & $55 / 68$ & $54 / 67$ & $46 / 67$ & $42 / 61$ & $45 / 66$ & $55 / 109$ & 64 \\
\hline $\begin{array}{r}\text { Correct } \\
\text { (Classified) Atypical nevus }(A N)\end{array}$ & $64 / 67$ & $65 / 70$ & $63 / 68$ & $53 / 63$ & $55 / 64$ & $53 / 61$ & $46 / 52$ & 73 \\
\hline $\begin{array}{r}\text { Correct } \\
\text { (Classified) Suspicious nevus }(\mathrm{SN})\end{array}$ & $25 / 26$ & $21 / 25$ & $23 / 27$ & $25 / 30$ & $26 / 35$ & $25 / 33$ & $22 / 24$ & 26 \\
\hline $\begin{array}{r}\text { Correct } \\
\text { (Classified) Benign nevus }(B N)\end{array}$ & $107 / 108$ & $107 / 107$ & $107 / 108$ & $107 / 110$ & $106 / 110$ & $107 / 110$ & $84 / 85$ & 107 \\
\hline $\left.\begin{array}{r}\text { SENSITIVITY \% } \\
{\left[M N_{\text {corr_classif }} / M_{\text {Reference }}\right]}\end{array}\right]$ & 92 & 86 & 84 & 72 & 66 & 70 & 86 & - \\
\hline $\begin{array}{r}\text { SPECIFICITY \% } \\
{\left[(\mathrm{AN}+\mathrm{SN}+\mathrm{BN})_{\text {corr_classif }} /\left(\text { Data- } \mathrm{MN}_{\text {Reference }}\right)\right]}\end{array}$ & 95 & 94 & 94 & 90 & 91 & 90 & 74 & - \\
\hline DIAGNOSTIC ACCURACY \% & 94 & 92 & 92 & 86 & 85 & 85 & 77 & - \\
\hline CLINICAL ACCURACY \% & 94 & 92 & 91 & 86 & 85 & 85 & 77 & - \\
\hline
\end{tabular}

As opposite behavior, the performance exhibited by the MS shows a good capability for the melanoma detection and low specificity, which adversely affects the overall diagnostic and clinical accuracy. Main motivation is represented by the slight over-estimation of both major and minor dermoscopic criteria with respect to the baseline. Anyway, a deeper analysis (which takes also into account the Low, Medium and High levels and associated weights computed for the detection reliability) has revealed a closer agreement between MS and the best results for the ED Group (examiner S.C.): rank correlation coefficients are computed as greater than 0.75 for all the dermoscopic structures, with the highest concordance existing for Blue-whitish Veil, Atypical Pigment Network and Irregular Streaks. This finding led the authors to further investigate the effects concerning the adoption of the distributed platform as clinical support for the nonexpert dermatologist. The NED Group' physicians were asked to re-examine the dermoscopic database at three months distance assisted by the MS (presentations of both the detection results corresponding to the single criterion and the overall lesion classification).

The results are summarized in Table 3: NED greatly beneficiated of MS assistance, with an improving in diagnostic sensitivity (from $+14 \%$ to $17 \%$ ) without compromising specificity and resulting in a gain in terms of diagnostic accuracy (from 2\% to 4\%). The Cohen's Kappa test also showed the statistical validity of above mentioned results, excluding the possibility that the variation in diagnostic accuracy between NED and NED + MS was purely casual. 
Table 3. Automatic Classification according to the Weighted Scoring System.

\begin{tabular}{|c|c|c|c|c|c|c|c|c|}
\hline & \multicolumn{8}{|c|}{ Examiner } \\
\hline & \multicolumn{3}{|c|}{ NED } & \multicolumn{3}{|c|}{ NED + MS } & \multirow{2}{*}{$\frac{\text { MS }}{\text { MS }}$} & \multirow{2}{*}{$\begin{array}{c}\text { Biopsy/ } \\
\text { Reference }\end{array}$} \\
\hline & C.C. & A.M. & F.C. & C.C. & A.M. & F.C. & & \\
\hline $\begin{array}{r}\text { Correct (Classified) } \\
\text { Melanoma }(M N)\end{array}$ & $46 / 67$ & $42 / 61$ & $45 / 66$ & $55 / 77$ & $53 / 75$ & $54 / 76$ & $55 / 109$ & 64 \\
\hline $\begin{array}{r}\text { Correct } / \\
\text { (Classified) Atypical nevus }(A N)\end{array}$ & $53 / 63$ & $55 / 64$ & $53 / 61$ & $52 / 58$ & $55 / 58$ & $53 / 57$ & $46 / 52$ & 73 \\
\hline $\begin{array}{r}\text { Correct } / \\
\text { (Classified) Suspicious nevus }(S N)\end{array}$ & $25 / 30$ & $26 / 35$ & $25 / 33$ & $24 / 27$ & $26 / 31$ & $24 / 30$ & $22 / 24$ & 26 \\
\hline $\begin{array}{r}\text { Correct } \\
\text { (Classified) Benign nevus }(B N)\end{array}$ & $107 / 110$ & $106 / 110$ & $107 / 110$ & $107 / 108$ & $106 / 106$ & $106 / 107$ & $84 / 85$ & 107 \\
\hline $\begin{array}{r}\text { SENSITIVITY \% } \\
{\left[\mathrm{MN}_{\text {corr_classif }} / \mathrm{MN}_{\text {Reference }}\right]} \\
\end{array}$ & 72 & 66 & 70 & 86 & 83 & 84 & 86 & - \\
\hline $\begin{array}{r}\text { SPECIFICITY \% } \\
{\left[(\mathbf{A N}+\mathrm{SN}+\mathrm{BN})_{\text {corr_classif }} /\left(\text { Data }-\mathrm{MN}_{\text {Reference }}\right)\right]}\end{array}$ & 90 & 91 & 90 & 89 & 91 & 89 & 74 & - \\
\hline DIAGNOSTIC ACCURACY \% & 86 & 85 & 85 & 88 & 89 & 88 & 77 & - \\
\hline CLINICAL ACCURACY \% & 86 & 85 & 85 & 88 & 89 & 88 & 77 & - \\
\hline
\end{tabular}

\section{Conclusions}

An image based measurement system implementing a well-known diagnostic method is disclosed for the automatic detection of melanomas as support to clinicians. The distributed architecture is able to receive and store digital ELM images captured by hand-held dermoscopy and smartphones, recognize automatically the skin lesion within the digital image, measure morphological and chromatic parameters, carry out a suitable classification for detecting the dermoscopic structures included in the Seven Point Checklist diagnostic method and finally provide a second opinion supporting the dermatologist for the clinical decision. Advanced techniques are introduced at different stages of the image processing pipeline, including the border detection, the extraction of lowlevel features and scoring of high order features. Focus has been devoted both to the reliability measurement of the intermediate results (in order to improve the lesion classification) and the performance validation of the measurement system (related to the clinical practice at dermatology department). The implementation of the automatic system on commercial components has revealed the feasibility of the proposed architecture. Although the automatic measurement and detection system did not outperform the diagnostic capabilities of expert dermatologists' group, it has allowed a significant improvement of sensitivity and overall diagnostic accuracy for dermatologists with low dermoscopic experience ( $<3$ years). Further research will be concerning both with the correlations existing between the dermoscopic structures as visually inspected by the expert dermatologists among the different classes of skin lesions and the management of the measurement uncertainty associated to the multiscale computation of the proposed algorithms for the low-level features in order to improve the automatic detection of the criteria included in the Seven Point Checklist. Finally, an investigation about the Decision Trees and other Machine Learning techniques will be carried out to suitably train and test a classifier according to the diagnostic capabilities of the expert dermatologists: the classifier will be integrated into the proposed lesion classification scheme in order to improve the clinical accuracy of the automatic system.

\section{References}

[1] Skin Cancer Foundation, "Skin Cancer Foundation Skin cancer facts", http://www.skincancer.org/SkinCancer-Facts/(2015).

[2] G.P. Guy, S.R. Machlin, D.U. Ekwueme, K.R. Yabroff, "Prevalence and costs of skin cancer treatment in the U.S., 2002-2006 and 2007-2011", Am. J. Prev. Med. 2014; 104(4): e69-e74, DOI: dx.doi.org/10.1016/j.amepre.2014.08.036.

[3] P. Mehta, K. Chand, D. Narayanswamy, D.G. Beetner, R. Zoughi, W.V. Stoecker, "Microwave reflectometry as a novel diagnostic tool for detection of skin cancers," Instrumentation and Measurement, IEEE Transactions on, Vol.55, no.4, pp.1309,1316, 2006. 
[4] G. Sforza, G. Castellano, S.A. Arika, R.W. LeAnder, R.J. Stanley, W.V Stoecker, J.R. Hagerty, "Using Adaptive Thresholding and Skewness Correction to Detect Gray Areas in Melanoma In Situ Images," Instrumentation and Measurement, IEEE Transactions on, Vol.61, no.7, pp.1839,1847, July 2012.

[5] S.M. Rajpara, A.P. Botello, J. Townend et alii, "Systematic Review of dermoscopy and digital dermoscopy/artificial intelligence for the diagnosis of melanoma", British Journal of Dermatology $\mathrm{N}^{\circ} 161$ : pp. 591-604, 2009.

[6] J. March, M. Hand, D. Grossman, "Practical application of new technologies for melanoma diagnosis: Part I. Noninvasive approaches", Journal of American Academy of dermatology, Vol. 72, $\mathrm{N}^{\circ} 6$, pp. 929-941, 2015.

[7] D. Piccolo, A. Ferrari, K. Peris et al., "Dermoscopic diagnosis by a trained clinician vs. a clinician with minimal dermoscopy training vs. a computer-aided diagnosis of 341 pigmented skin lesions: a comparative study", British Jour. of Dermat., 147:481-486, 2002.

[8] A. Blum, H. Luedtke, U. Ellwanger, R. Schwabe, G. Rassner, C. Garbe,"Digital image analysis for diagnosis of cutaneous melanoma. Development of a highly effective computer algorithm based on analysis of 837 melanocytic lesions", British Journal of Dermatology 2004, Vol. 151, N 5, pp. 1029-1038.

[9] M.J. Vasconcelos, L. Rosado, M. Ferreira, "A New Risk Assessment Methodology for Dermoscopic Skin Lesion Images based on the independent analysis of each $A B C D$ rule criterion", Proc. of 2015 IEEE International Workshop on Medical Measurement and Applications, (MeMeA'15); Torino, Italy; 2015, pp. 570-575.

[10] G. Di Leo, A. Paolillo, P. Sommella, G. Fabbrocini, O. Rescigno, "A software tool for the diagnosis of melanomas automatic implementation of the 7-point check list method", 2010 IEEE International Instrumentation and Measurement Technology Conference, I2MTC 2010, pp. 886-891, 2010.

[11] G. Di Leo, C. Liguori, A. Paolillo, P. Sommella, "A Web-based Application for Dermoscopic Measurements and Learning", Proc. of 2015 IEEE International Workshop on Medical Measurement and Applications, (MeMeA'15); pp. 279-284, 2005.

[12] http:77www.i3-dermoscopy.it

[13] G.Argenziano, H. P. Soyer, V. De Giorgi et alii, "Interactive Atlas of Dermoscopy", EDRA Medical Publishing \& New Media, Milan, Italy, 2002.

[14] D. Capriglione, L. Ferrigno, C. Liguori, A. Paolillo, P. Sommella, and F. Tortorella, "Digital processing of diagnostic images," in Advances in Biomedical Sensing, Measurements, Instrumentation and Systems (Lecture Notes in Electrical Engineering), vol. 55, S. C. Mukhopadhyay and A. Lay-Ekuakille, Eds. New York, NY, USA: Springer-Verlag, 2010, p. 450.

[15] P. Schmid, "Lesion Detection in Dermatoscopic Images Using Anisotropic Diffusion and Morphological Flooding", IEEE Image Processing 1999, pp. 449-453.

[16] M. E. Celebi, H. A. Kingravi et alii., "Border detection in dermoscopy images using statistical region merging", Skin Research and Technology 2008 Vol 13, $\mathrm{N}^{\circ} 4$, pp. 347-353.

[17] F. Kurugollu, B. Sankur, A. E. Harmanci, "Color image segmentation using histogram multithresholding and fusion", Imaging and Vision Computing $\mathrm{N}^{\circ} 19$, pp. 915-928, 2001.

[18] G. Di Leo, C. Liguori, A. Paolillo, P. Sommella, "An Improved procedure for the automatic detection of dermo-scopic structures in digital ELM images of skin lesion", IEEE Conference on Virtual Environments, Human-Computer Interfaces and Measurement Systems VECIMS 2008, pp. 190-194 2008.

[19] W. Xin; N.D. Georganas, E.M. Petriu, "Fabric Texture Analysis Using Computer Vision Techniques," Instrumentation and Measurement, IEEE Transactions on , vol.60, no.1, pp.44,56, Jan. 2011.

[20] M. Sadeghi, M. Razmara, P. Wighton, T. K. Lee and M. S. Atkins, "A Novel Method for Detection of Pigment Network in Dermoscopic Images Using Graphs", Computerized Medi-cal Imaging and Graphics, Vol. 35, N 2, pp. 137-143, 2011.

[21] A. Frangi, W.J. Niessen, K.L. Vincken, and M.A.Viergever, "Multiscale vessel enhancement filtering", MICCAI, pages 130-137, 1998.

[22] P. Wighton, T. K. Lee, H. Lui, D. I. McLean and M. S. Atkins, "Generalizing Common Tasks in Automated Skin Lesion Diagnosis", IEEE Transactions on Information Technology in Biomedicine, Vol. 15, $\mathrm{N}^{\circ} 4$, pp. 622-629, 2011

[23] T.K. Abdel-Galil, R.M. Sharkawy, M.M.A. Salama, R. Bartnikas, "Partial discharge pattern classification using the fuzzy decision tree approach," Instrumentation and Measurement, IEEE Transactions on , vol.54, no.6, pp.2258,2263, 2005.

[24] M.S. Manikandan, S.R. Samantaray, I. Kamwa, "Detection and Classification of Power Quality Disturbances Using Sparse Signal Decomposition on Hybrid Dictionaries," Instrumentation and Measurement, IEEE Transactions on , vol.64, no.1, pp. 27,38 , Jan. 2015

[25] O. Debeir, C. Decaestecker, "Computer-Assisted Analysis of Epiluminescence Microscopy Images of Pigmented Skin Lesions", Cytometry, $\mathrm{N}^{\circ} 37$, pp. 255266, 1999

[26] M. Sadeghi, T. K. Lee, D. I. McLean, H. Lui and M. S. Atkins, "Detection and Analysis of Irregular Streaks in Dermoscopic Images of Skin Lesions", IEEE Transactions on Medical Imaging, 2013.

[27] N .Landwehr, M. Hall, E. Frank, "Logistic Model Trees",14th European Conference on Ma-chine Learning, 2003

[28] J. Kirk, "Count loops in a network", http://www.mathworks.com/matlabcentral/fx_files/ 10722/1/content/html/run_loops_html.html (2007), accessed 12 May 2009.

[29] V. De Vita, G. Di Leo, G. Fabbrocini, C. Liguori, A. Paolillo, P.Sommella,"Statistical image processing for the detection of dermoscopic criteria", 18th IMEKO TC4 Symposium on Measurement of Electrical Quantities, Part of Metrologia, pp. 128-133, 2011. 